\title{
Scar-Free Healing of Wounds Resulting Commonly from Domestic Accidents
}

\section{Wan Keung Raymond Wong*, Ka Lun Ng and Cheuk Yin Lai}

Institutional affiliation: Division of Life Science, The Hong Kong University of Science and Technology, Clear Water Bay, Kowloon, Hong Kong, China

\section{*Corresponding authors}

Wong W.K.R, Division of Life Science, The Hong Kong University of Science and Technology, Clear Water Bay, Kowloon, Hong Kong, China.

Submitted: 17 March2021;Accepted: 25 March 2021;Published: 05 Apr 2021

Citation: Wan Keung Raymond Wong, Ka Lun Ng, Cheuk Yin Lai (2021) Scar-Free Healing of Wounds Resulting Commonly from Domestic Accidents. Medical \& Clinical Research 6(4): 531-535.

\begin{abstract}
Scalds and cuts are common causes of childhood injuries at home. Although the impairments are often non-fatal, timely and effective treatments for them may not only help prevent infections, but also facilitate faster recovery from the injuries and scarless wound healing. Skin growth factors including the 53-amino acid human epidermal growth factor (EGF) and the 146-amino acid human basic fibroblast growth factor (bFGF) have been shown to play important physiological functions in promoting the growth and regeneration of our skin cells. Moreover, they have been employed individually to enhance the healing process of various types of wound. The specific bioactivities exhibited by EGF and bFGF in the epidermal and dermal layers, respectively, strongly support the notion that they may function cooperatively in wound healing. In this communication, making use of our own recombinant EGF ( $r E G F)$ and bFGF (rbFGF) products, which share the same primary structures with their native counterparts, we present research findings to demonstrate that $r E G F$ and $r b F G F$ work collaboratively to promote healing of various types of wound, including scalds, punctures and lacerations. More importantly, despite the severity of the injuries, subsequent to their treatments with $r E G F$ and $r b F G F$, the healed wounds were virtually scar-free and devoid of the formation of an outgrowth scar, a keloid scar.
\end{abstract}

Keywords: Authentic Human Epidermal Growth Factor; Authentic Human Basic Fibroblast Growth Factor; Scar-Free Healing; Scalds; Punctures; Lacerations

\section{Abbreviations}

EGF: Human Epidermal Growth Factor;

bFGF: Human Basic Fibroblast Growth Factor;

rEGF: recombinant EGF;

rbFGF: recombinant bFGF;

EP: Enhanced Platinum cream

\section{Introduction}

Since the isolation and characterization of human epidermal growth factor (EGF), a 53-amino acid polypeptide, was achieved by Cohen and Carpenter in 1975, a great number of articles reporting the application of this small skin growth factor to various wound treatments have been published [1-4]. In the early days, findings regarding the efficacy and safety of EGF for use as an active ingredient in medical and skin care applications were controversial $[5,6]$. However, it was later shown that the inconsistent and contentious issues stemmed from the employment of EGF derivatives or variants, which possessed likely unauthentic structures that were different from the 53 residue authentic EGF [7]. Moreover, neither the sources nor the exact compositions of the counterfeit materials were reported, thus making it difficult to understand the causes of inefficacy or undesirable effects [7].

Our laboratory has been involved in the engineering of efficient bacterial platforms for cost-effective production of valuable recombinant proteins, among which recombinant EGF (rEGF) represents a good example that has been developed for over 2 decades $[8,9]$. The availability of rEGF has not only helped to reduce dramatically the market price of the factor, but has also served as an effective means for the treatments of a wide spectrum of skin diseases including diabetic foot ulcers, bedsores and acute allergies such as Steven Johnson syndrome [10-13].

Realizing that it might take longer durations for rEGF to heal deeper or more serious wounds, we explored the feasibility of producing recombinant basic fibroblast growth factor (rbFGF), a 146 residue polypeptide, of which the native counterpart had been shown to play a crucial role in the formation of collagen and elastin in the dermal layer of skin [14]. In 2013, we pioneered the expression of authentic rbFGF employing various host systems [15-20]. It was then applied topically in conjunction with rEGF to treat puncture wounds resulting from micro-needling treatment 
in skin care operations. The outcomes turned out to be highly effective and the wounds healed speedily and smoothly to result in apparently scar-free skin regeneration [12].

Scalds and various kinds of physical impairments, e.g. head injuries due to slip or trip and fall accidents are a common cause of self-inflicted injuries occurring among teenagers or youngsters at home. Although most of these accidents are non-fatal, delayed treatment may result in the formation of scar during the healing process. In this communication, we provide successful examples to illustrate the high effectiveness of rEGF and rbFGF in treating domestic injuries inflicted by the victims. Attractively, complete healing was not only achieved within short durations of time, but also with scar-free regeneration of the skin tissue.

\section{Materials and Methods}

\section{Sources of rEGF and rbFGF}

The production and purification of authentic rEGF and rbFGF achieved in our laboratory was published previously [8, 9, 15-21].

\section{Cream Product Employed for Wound Healing}

The final cream product named Enhanced Platinum cream (EP) containing $0.04 \%(\mathrm{w} / \mathrm{w})$ authentic $\mathrm{rEGF}$ and $0.01 \%$ authentic
rbFGF (w/w) was procured from Gene-vinate Limited (GVN): www.gene-vinate.com [22].

\section{Treatment of wounds}

A protocol for the topical treatment of wounds was established previously [23]. In brief, an injured region was cleansed thoroughly with diluted Dettol (1:20) or hydrogen peroxide, followed by applying a thin layer of EP cream on the disinfected area. A gauze pad was then put on top of the treated surface. The process was done once (at night) or twice daily depending on the severity of the impairment.

\section{Results and discussion}

Scalded Wounds: Case 1

The left side of the face and chin of a toddler girl living in Anhui, China, was accidentally scalded by hot water and around $50 \%$ of her left face was affected, leading to the formation of a large blister on the injured skin (Figure 1). The girl was sent to consult a medical doctor immediately and a commercial "EGF" cream was then prescribed to treat the wound. Unfortunately, despite being treated for 3 days, there was no sign of improvement. The girl's mother was so worried that the symptoms might get worse and result in scar formation even though the wound was finally healed.

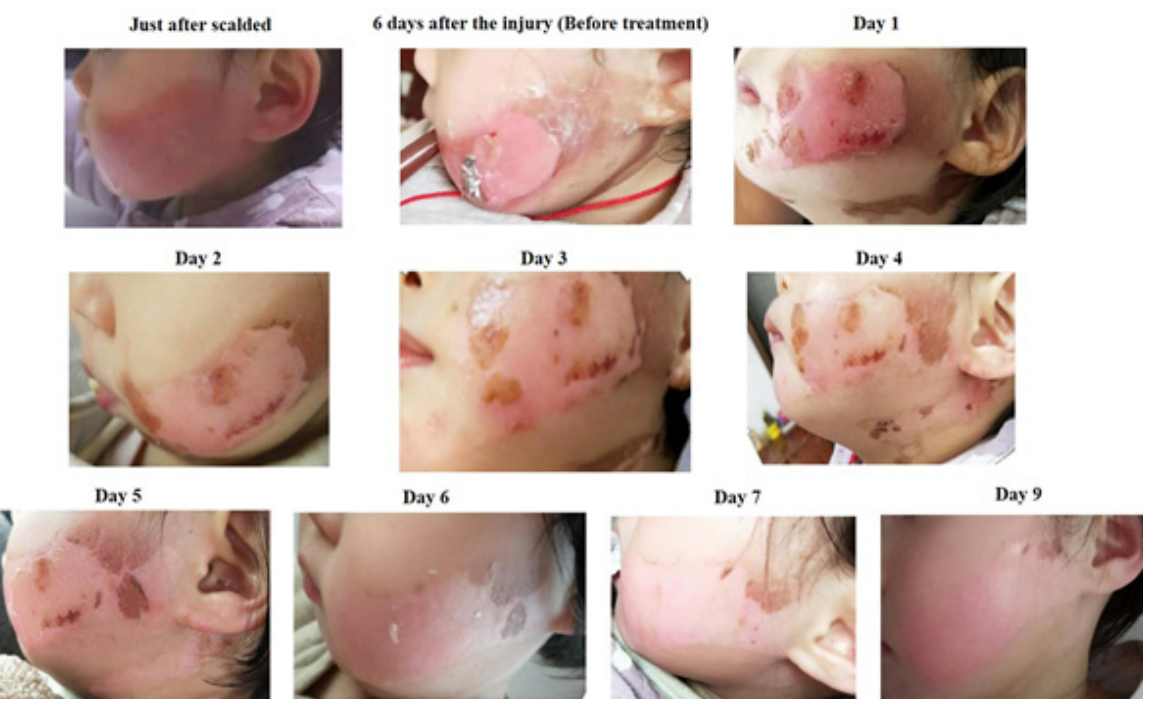

Figure 1: Treatment of scalded wound with EP: Case 1.

The left side of the face and chin of a toddler girl was scalded by hot water. EP treatment commenced 6 days after the injury (Day 1), healing signs including bursting of the blister, dehydration of dead skin cells and relief of pain were observed after 4 days of treatment (Day 4). Pronounced recovery was observed after 6 days of treatment (Day 6), whereas complete scar-free healing of the wound was shown after 9 days of treatment (Day 9).

With the assistance of a friend staying in Hong Kong, an order of Enhanced Platinum cream (EP; Materials and methods) was placed and it took 3 days for the delivery to arrive at the destination. Thus, the victim was not treated by EP until the accident had occurred for 6 days (Figure 1). Despite the delayed treatment, the healing progressed well and showed significant improvements: bursting of the blister, dehydration and exfoliation of dead skin cells, and most importantly, relief of pain, which were observed in the first 4 days after the commencement of treatment (Figure 1). In the next 5-9 days, the skin color was returning to normal and epithelialization was observed without any sign of scar formation (Figure 1). All these beneficial effects were concluded to be attributable to the miraculous cooperativity between rEGF and rbFGF in wound healing.

\section{Scalded wounds: Case 2}

A local 6-year-old boy was accidentally scalded by a bowl of hot soup while he was playing at home. A large area covering the upper part of his left back, the nape and head regions was severely injured (Figure 2). Although initial care was received swiftly from a local clinic and follow-up treatment with an antibiotic ointment was done as advised, the prescribed regimen did not work well. 
The boy still suffered intense pain in his upper back and neck for the next couple of days. The boy's family then approached us for assistance on the 3rd day after the accident. The first thing we did was to conduct thorough cleansing of the wound using a diluted Dettol solution according to the protocol published previously [23]. After air drying of the disinfected area, EP was employed and applied topically to the wound according to the details mentioned in Case 1 and published previously [23]. The wound was treated two times with EP on the 1st day. On the 2nd day, encouragingly, reminiscent of the findings observed in Case 1, the blister dried and flattened out, and the outcomes markedly alleviated the injury and pain experienced by the victim. Over the next few days, despite the treatment being administered only once per day, EP was shown to be highly effective in promoting the regeneration of damaged skin. After 8th day of treatment, the wound was completely healed (Figure 2), and the healed skin was expected to regain a normal tone eventually.

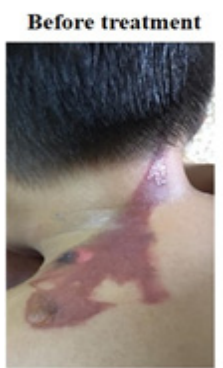

Day 4

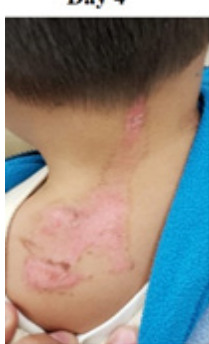

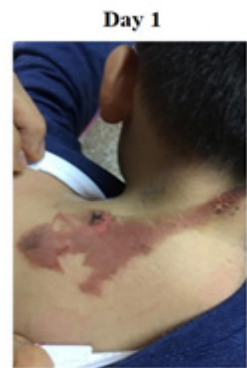

Day 6

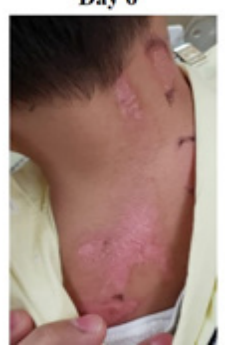

Day 2

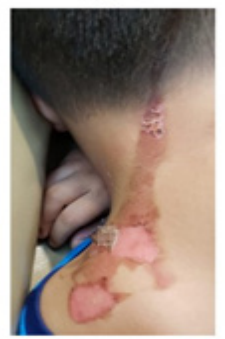

Day 8

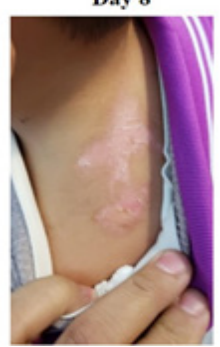

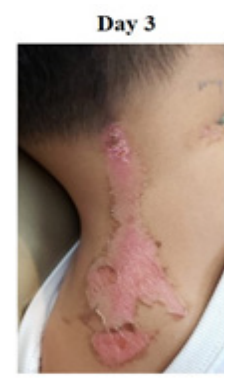

Day 9

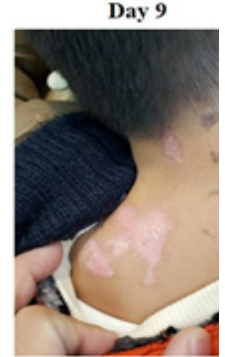

Figure 2: Treatment of scalded wound with EP: Case 2.

A local 6-year-old boy was scalded by hot soup (Before treatment). EP treatment of the wound was not administered until 3 days after the injury (Day 1). Healing signs including flattening and drying of the blister and regaining a natural skin tone were observed in the next few days of treatment. The wound healed completely after 9 days of treatment.

\section{Puncture Wounds}

A local toddler girl suffered a deep gash on her right forehead which was punctured by a sharp object when she tripped and fell while playing. She was sent to the hospital with the incision stitched up without delay. Despite successfully being treated medically (Figure 3), the girl's parents were so worried that a scar would leave behind after the wound was healed. They sought advice from us and we immediately recommended them to apply EP (twice daily) to the sutured wound, based on the fact that bFGF is capable of promoting both epithelial and dermal repair and more importantly, inhibiting scar formation [24]. The treatment regimen worked exceptionally well and on the 8th day, the stitches were removed. Although there was a small dent left behind, it was deemed that this minor defect could be decently recovered. Thus, a skin care product, Enhanced Essence, which contained both rEGF and rbFGF at a reduced concentration $(0.0003 \%$, w/w), was recommended for use to treat the freshly closed incision [22]. After 60 days of treating the dent with Enhanced Essence, remarkably, the size of it had been reduced to a virtually unnoticeable mark (Figure 3), of which the girl's parents were very pleased with the remarkable outcome.
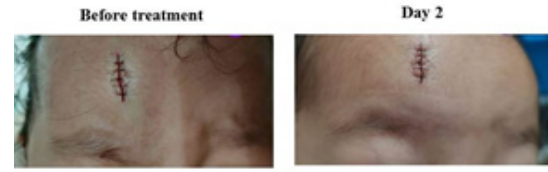

Day 8

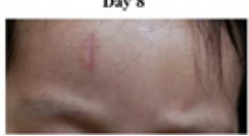

Day 10

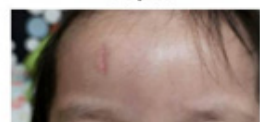

Day 4

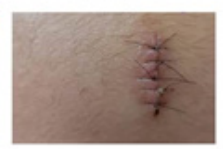

Day 32

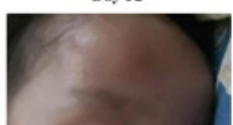

Day 6

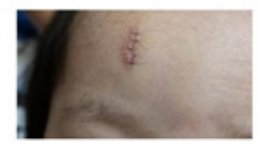

Day 60

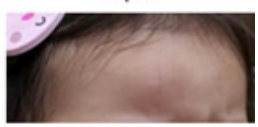

Figure 3: Treatment of a puncture wound with EP.

The forehead of a local toddler girl was punctured by a sharp object. The incision was stitched up, followed by treatment with EP twice daily. The wound healed readily and after 8 days of treatment, the stitches were removed, and remarkably, only a small dent left behind 
(Day 8). Subsequently, Enhance Essence, which contained both rEGF and rbFGF at a reduced concentration $(0.0003 \%$, w/w) [22], was continuously employed to treat the dent. After 52 days of treatment (Day 60), the dent turned into a virtually unnoticeable mark.

\section{Lacerations}

A local teenage boy suffered a knife wound to his upper lip (Figure 4) while having a fight with another young male. Despite being sent immediately for treatment and the wound being diagnosed to be non-life threatening, the victim worried so much that an ugly scar would stay behind even though the incision was completely healed. Based on our experience received from treating the puncture wound described above, the teenager was advised to undertake EP treatment soon after he had received sutures for his lip laceration. Incredibly, the regeneration process worked so well that by the time the stitches were removed (on the 10th day), only a slight dent remained (Figure 4). Moreover, the dint became virtually invisible subsequent to 8 more days of EP treatment (Figure 4).
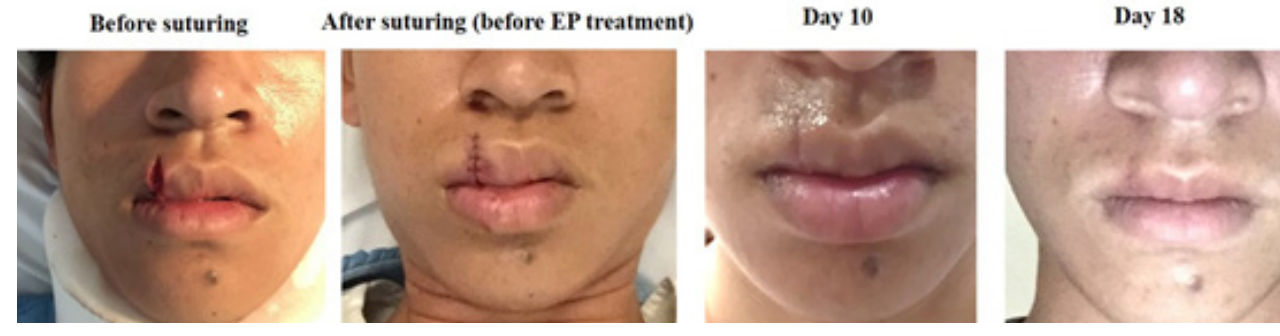

Figure 4: Treatment of a laceration with EP.

A teenage boy suffered a knife wound to his upper lip and received stitches at a hospital soon after the incident. Soon after the surgery, the wound also received EP treatment. The stitches were removed on the 10th day, and pleasingly, only a small dent remained behind. The dint became virtually invisible after 8 more days of EP treatment.

\section{Conclusion}

Impaired wound healing may not only result in complications including infections and delayed healing, but may also lead to an undesirable consequence of scar formation. Our research findings show that rEGF and rbFGF work cooperatively and effectively in the healing process in sloughing off damaged skin cells, regenerating new skin cells and tissues, as well as preventing scar formation.

\section{Acknowledgement}

This study was supported by Research Contract No.1617219-0 managed by the R\&D Branch, HKUST.

\section{References}

1. Cohen S, Carpenter G (1975) Human epidermal growth factor: isolation and chemical and biological properties. Proc Natl Acad Sci USA 72: 1317-1321.

2. Franklin JD, Lynch JB (1979) Effects of topical applications of epidermal growth factor on wound healing. Experimental study on rabbit ears. Plast Reconstr Surg 64: 766-770.

3. Niall M, Ryan GB, O'Brien BM (1982) The effect of epidermal growth factor on wound healing in mice. J Surg Res 33: 164-169.

4. Arturson G (1984) Epidermal growth factor in the healing of corneal wounds, epidermal wounds and partial-thickness scalds. A controlled animal study. Scand J Plast Reconstr Surg 18: 33-37.

5. Greaves MW (1980) Lack of effect of topically applied epidermal growth factor (EGF) on epidermal growth in man in vivo. Clin Exp Dermatol 5: 101-103.

6. Falanga V, Eaglstein WH, Bucalo B, Katz MH, Harris B, et al. (1992) Topical use of human recombinant epidermal growth factor (h-EGF) in venous ulcers. J Dermatol Surg Oncol 18: 604-606.

7. Wong WKR, Ng KL, Lam CC, Hu XH, Lai NCY, et al. (2017)
Review article: reasons for underrating the potential of human epidermal growth factor in medical applications. J Anal Pharm Res 4: 00101.

8. Huang RC, Lam E, Chen YH, Hackett J, Lam TL, et al. (1999) Human epidermal growth factor excreted by recombinant Escherichia coli K-12 has the correct N-terminus and is fully bioactive. Process biochemistry 35: 1-5.

9. Sivakesava S, Xu ZN, Chen YH, Hackett J, Huang RC, et al. (1999) Production of excreted human epidermal growth factor (hEGF) by an efficient recombinant Escherichia coli system. Process biochemistry 34: 893-900.

10. Tsang MW, Wong WKR, Hung CS, Lai KM, Tang W, et al. (2003) Human epidermal growth factor enhances healing of diabetic foot ulcers. Diabetes care 26: 1856-1861

11. Tsang MW, Tsang KY, Wong WKR (2004) The use of recombinant human epidermal growth factor (rhEGF) in a gentleman with drug induced Steven Johnson syndrome. Dermatol Online J 10: 25.

12. Wong WKR, Ng KL, Hu XH (2018) Authentic human epidermal growth factor: a panacea for wound healing. EC Endocrinology and Metabolic Research 3: 138-146.

13. Wong WKR, Ng KL, Lam CC, Wang H, Hu XH, et al. (2019) The importance of authentic human epidermal growth factor in offering effective treatments for hard-to- heal wounds. EC Diabetes and Metabolic Research 3: 4.

14. Bikfalvi A, Klein S, Pintucci G, Rifkin DB (1997) Biological roles of fibroblast growth factor-2. Endocr Rev 18: 26-45.

15. Kwong KW, Wong WKR (2013) A revolutionary approach facilitating co-expression of authentic human epidermal growth factor and basic fibroblast growth factor in both cytoplasm and culture medium of Escherichia coli. Appl Microbiol Biotechnol 97: 9071-9080.

16. Hu XH, Lai CYN, Sivakumar T, Wang H, Ng KL, et al. (2018) Novel strategy for expression of authentic and bioactive human basic fibroblast growth factor in Bacillus subtilis. Appl 
Microbiol Biotechnol 102: 7061-7069.

17. Wong WKR (2020) Means and method for expression of authentic human epidermal growth factor and/or basic fibroblast growth factor in cytoplasm and/or Culture Medium of Escherichia coli. Chinese Patent No.CN 105143451 B

18. Wong WKR (2018) Means and method for expression of authentic human epidermal growth factor and/or basic fibroblast growth factor in cytoplasm and/or culture medium of Escherichia coli. Japan Patent No 6404901.

19. Wong WKR (2017) Means and method for expression of authentic human epidermal growth factor and/or basic fibroblast growth factor in cytoplasm and/or culture medium of Escherichia coli. Hong Kong short-term patent No HK1219200

20. Wong WKR (2017) Means and method for expression of authentic human epidermal growth factor and/or basic fibroblast growth factor in cytoplasm and/or culture medium of Escherichia coli. US Patent No 9: 580, 484 B2

21. Sivakumar T, Lai CYN, Lam CC, Hu X, Wang H, et al. (2018) Purification of authentic human basic fibroblast growth factor expressed in both the cytoplasm and culture medium of Escherichia coli. Int J Chromatogr Sep Tech: IJCST-115.

22. Gene-vinate Limited website: www.gene-vinate.com.

23. Wong WKR (2015) Effective treatment of an unhealed incision of a diabetic patient with recombinant human epidermal growth factor. Mod Chem appl 3: 166.

24. Keisuke O, Ruka H, Noriko A-H, Yoshiaki S, Kazuo K (2013) Wound treatment using growth factors. Modern Plastic Surgery 3: 108-112.
Copyright: (C2021 Wong W.K.R, et al. This is an open-access article distributed under the terms of the Creative Commons Attribution License, which permits unrestricted use, distribution, and reproduction in any medium, provided the original author and source are credited. 